\title{
An IoT-based Prediction Technique for Efficient Energy Consumption in Buildings
}

\author{
Shidrokh Goudarzi, Member, IEEE, Mohammad Hossein Anisi, Senior Member, IEEE, Seyed Ahmad \\ Soleymani, Member, IEEE, Masri Ayob, Senior Member, IEEE Sherali Zeadally, Senior Member, IEEE
}

\begin{abstract}
Today, there is a crucial need for precise monitoring and prediction of energy consumption at the building level using the latest technologies including Internet of Things (IoT) and data analytics to determine and enhance energy usage. Datadriven models could be used for energy consumption prediction. However, due to high non-linearity between the inputs and outputs of energy consumption prediction models, these models need improvement in terms of accuracy and robustness. Therefore, this work aims to predict energy usage for the optimum outline of building-extensive energy distribution strategies based on a lightweight IoT monitoring framework. To calculate accurate energy consumption, an enhanced hybrid model was developed based on Auto-Regressive Integrated Moving Average (ARIMA) and Imperialist Competitive Algorithm (ICA). The parameters of the ARIMA model were optimized by adapting the ICA technique that improved fitting accuracy while preventing over-fitting on the acquired data. Then, Exponentially Weighted Moving Average (EWMA) was applied to monitor the predicted values. The proposed AIK-EWMA hybrid model was assessed based on the actual power consumption data and validated using mathematical tests. As compared to previous works, the findings revealed that the hybrid model could accurately predict power consumption for green building automation applications.
\end{abstract}

Index Terms-Artificial intelligence, auto-regressive integrated moving average, imperialist competitive algorithm, building energy consumption, prediction.

\section{INTRODUCTION}

A green building is constructed with a number of strategies to save different resources, reduce waste and life cycle costs, and create an environmental-friendly place for people. [1]-[5]. This requirement is particularly important for multi-functional community and buildings that tend to be used continuously for various purposes.

Effective energy management in green buildings can decrease their operating expenses [6]. This can be achieved by evaluating the energy consumed by end consumers, such as lighting and electrical equipment, and reducing the energy consumed for a separate group or an end-user group. Recent advances in IoT technologies have opened up new ideas

S. Goudarzi is with Centre for Artificial Intelligent (CAIT), Universiti Kebangsaan Malaysia, 43600 Bangi, Malaysia (e-mail: shidrokh@ukm.edu.my).

M. H. Anisi is with the School of Computer Science and Electronic Engineering, University of Essex, Colchester CO4 3SQ, UK (e-mail: m.anisi@essex.ac.uk).

S. A. Soleymani is with the School of Computing, Faculty of Engineering, Universiti Teknologi Malaysia, Johor, Malaysia (e-mail: asseyed4@live.utm.my).

M. Ayob is with the Faculty of Information Science and Technology, University Kebangsaan Malaysia (e-mail: masri@ukm.edu.my).

S. Zeadally is with the College of Communication and Information University of Kentucky, USA (e-mail: szeadally@uky.edu). for real-time collection of the required data [7]. Monitoring each electricity outlet in a house using IoT and modifying how the electricity is consumed can lead to efficient energy consumption. For instance, Tao [8] proposed an energyefficient scheduling mechanism for cloud service management. The energy consumption data produced throughout a product life-cycle can be obtained dynamically and in real-time by adopting IoT technologies.

In addition, various energy management methods have been proposed to control energy consumption [9], [10]. Energy consumption prediction approaches based on real-time monitoring have been used to reduce the cost of energy consumption. These methods can minimize the total energy consumption by predicting buildings' energy usage along with the comfort level of customers for different smart city applications [11], [12]. By using energy prediction methods, customers can reduce their electricity usage over critical peak hours. They can shift their usage from peak to off-peak hours, resulting in changes to the consumer's electricity usage pattern [13]. The Auto-Regressive Integrated Moving Average (ARIMA) model [14], [15] has been used in various scientific fields for different sectors, such as energy sector [13], daily number of COVID-19 cases prediction [14], vehicular traffic flow forecast [15], smart homes [16], and sales prediction [17]. It is, therefore, well suited for energy consumption prediction and can be adapted for monthly consumption [18]. To predict long-term energy consumption, in [19], multiple regression analysis methods are utilized, while the decomposition method is described in [20]. Moreover, the Exponentially Weighted Moving Average (EWMA) [21] method is a useful and easyto-understand method for the prediction of future demand. EWMA is a popular prediction technique in the industry with minimal computer storage requirements. By constantly controlling the consumption of sensors and control actuators, the efficient energy use of residential places can be obtained. This data can enhance the usefulness and functionality of the vast multi-functional consumer spaces developed to create operative structures. These tools help obtain data regarding energy consumption. This data can be used to produce usage patterns that are essential to manage and optimize the total energy use of the green building.

The main contributions of this paper are threefold:

- We proposed a prediction technique for efficient energy consumption in buildings. In the proposed technique, the ARIMA model's parameters are optimized using a hybrid Imperialist Competitive Algorithm (ICA) algorithm.

- We optimized the parameters by employing ICA to gen- 
erate individuals' initial population (countries) and form basic ICA components. The ICA processes the computation load and optimizes the data modeling performance of the ARIMA.

- We used the EWMA model to monitor and detect small failures in energy consumption prediction. The predicted data are monitored by an EWMA control chart as a prior analytical prediction model, demonstrating that this approach provides better accuracy over the existing analytical prediction method.

In this paper, the proposed building energy prediction system developed based on AIK-EWMA hybrid model, as a green approach, allows each building to predict the electricity consumption. We evaluate the models using popular mathematical tests and the actual power consumption data captured by an IoT monitoring system deployed to monitor large prayer room and library building which contains several assets that consume energy.

The remainder of this paper is organized as follows: Section 2 presents related works on prediction techniques for IoTs. Section 3 presents our proposed framework and core modeling procedure. Section 4 presents a comparison of our approach against state-of-the-art methods. Section 5, presents two mathematical predictive accuracy testing methods. Finally, we make some concluding remarks in Section 6.

\section{RELATED WORK}

Several approaches [4], [5] have been used to model the forecasting of energy consumption. Moreover, different techniques can be utilized for simulation ranging from simple regression to physical principle-based models. The Multiple Linear Regression (MLR) and ARIMA methods are normally used as performance benchmarks. Linear regression analysis is widely performed using MLR, and forecasting time series data are typically analyzed by ARIMA. The three parameters, $p, q$, and $r$ usually define the ARIMA model and they are the number of differences, the number of auto-regressive terms, and the number of affecting average terms, respectively. A comprehensive description of the ARIMA model is described in [22], [23]. Originally, the ARIMA technique was proposed by Box-Jenkins [24] and it has been used in different applications to successfully estimate economic, marketing, and social subjects. As previously mentioned, the ARIMA technique has a weakness which is its low precision on non-stationary data prediction. To eliminate this shortcoming, the PSO algorithm proposed by Eberhart and Kennedy [25] was utilized to improve the ARIMA model [22]. The most popular model in the area of building energy could be data-driven model because of its proper prediction performance and small processing time. The stacking model [26], as a data-driven model, was proposed to predict energy consumption of buildings. The stacking model [26] uses the features of a number of base prediction method and merge the features to "meta-features". This guarantees that the datasets could be observed by the final model from divergent structural and spatial angles.

Additionally, the ARIMA model can discover the features of the energy data by statically adjusting the parameters. Numerous methods had been evaluated by Suganthi and Samuel in [27] regarding the energy prediction area and they proposed two models. They explored traditional forecasting techniques [28], including econometrics models, regression, time series, and ARIMA. They reviewed soft computing methods such as support vector regression models, fuzzy logic, and Genetic Algorithm (GA), a neural network for predicting national and regional energy demand. The authors found that ARIMA models work efficiently with neural systems and other soft computing approaches to improve the accuracy of predictions of energy usage. Additionally, there are many challenges in the load forecasting. For example, it is difficult to process big volumes of data provided by smart meters, and detect the domination factors that are most related to the electricity load [19].

In [29], the authors proposed an ARIMA-based model to forecast the peak loads in a short-term (i.e. hourly). The prediction data showed that the improved ARIMA, in terms of prediction models, resulted in better outcomes in terms of accurate forecasting of daily peak load than the original ARIMA and yielded a Mean Absolute Percentage Error (MAPE) up to $1.98 \%$ for the public holiday case study presented.

Generally, the hybrid prediction model uses an ARIMA method [30]-[32] as a linear method for linear component prediction and applies a nonlinear model for other component prediction in time series. ARIMA models involving one variable because the time-series history is used to present the variables functionality with the preceding random variant. The ARIMA model is comprised of three modules: AR, IA, and MA. The basis of the model is a linear arrangement of the $\operatorname{AR}(p)$ and $\operatorname{MA}(q)$ called Auto-Regressive Integrated MovingAverage (ARIMA). The combination of AR and MA processes is ARIMA $(p, q)$. In general, in the ARIMA $(p, d, q)$ model, $p$ is defined as an order of AR process, and $q$ is defined as an order of MA process. The model describes the time series as follows [24]:

$$
\phi(B) \nabla^{d} x_{t}=\theta(B) e_{t}
$$

where $x_{t}$ and $e_{t}$ represent energy consumption and random error at time $t$, respectively. $B$ denotes an operator defined by $B x_{t}=x_{t-1}$, and this backward shift operator is associated with $\nabla ; d$ denotes the order of differencing; $\nabla=1-B, \nabla^{d}=$ $(1-B)^{d}$.

The two main operatives of $\phi(B)$ and $\theta(B)$ are AutoRegressive (AR) and Moving Averages (MA) operatives with orders $p$ and $q$, which are defined individually as follows [24]:

$$
\begin{gathered}
\phi(B)=1-\phi_{1} B-\phi_{2} B^{2}-\cdots-\phi_{p} B^{p} \\
\theta(B)=1-\theta_{1} B-\theta_{2} B^{2}-\cdots-\theta_{q} B^{q}
\end{gathered}
$$

where $\phi_{1}, \phi_{2}, \phi_{3}, \cdots, \phi_{p}$ are the auto-regressive coefficients, and $\theta_{1}, \theta_{2}, \theta_{3}, \cdots, \theta_{q}$ are the moving average quantities. The linear transmission function of the noise series can present the time series $x_{t}$ as follows:

$$
x_{t}=\mu+\phi(B) e_{t}
$$




$$
\phi(B)=1+\phi_{1} B+\phi_{2} B^{2}+\cdots
$$

where $\phi(B)$ is computed as $\phi(B)=\theta(B) / \phi(B)$. Generally, to create the ARIMA model, three phases are performed. The first step is referred to as model identification. In this step, the data is fixed considering their high importance in the ARIMA prediction model. To eliminate the variations in the data and stabilize the variance, differencing is typically applied to the data [33], and $d$ parameter is calculated. The second phase is parameter estimation, in which Schwarz's Bayesian Information Criterion (BIC) and Akaike's Information Criterion (AIC) [34] are reduced by approximation of the model parameters wherein the maximum log-probability of the model is calculated. BIC is operationally the same as AIC. However, a penalty expression is included that is related to the sample size. The third phase involves the assessment of accuracy and the error stationary of the model. The best model is selected based on estimating some error performance actions, including the Root Mean Square Error (RMSE) and Mean Absolute Error (MAE) [34].

\section{Problem Formulation}

We defined the energy consumption in the green building as a time series problem. It is associated with a sequence of observations over time as $a_{i}=\left\{a_{1}, a_{2}, \cdots\right\}$ where each observation in $a_{i} \in R$ matches to a particular time step $i$. The predicted data is defined as $b_{i} \in R$ and $b_{i}$ is the predicted values of energy consumption. The hybrid prediction model is considered as an optimization problem, where a predictor function $p$ predicts a next step energy consumption value yield as $b_{i+1}$ for upcoming time step predictions. The multiple steps of prediction $(\tau)$ is computed based on sliding window method and it is defined as follows:

$$
b_{i+\tau}=p\left(a_{i+\tau}, a_{i-1+\tau}, \cdots, a_{i-w+\tau}\right)
$$

where the window size is $w$. If the size of window is $w=1$, the prediction function will be $b_{i+1}=p\left(a_{i}\right)$. The objective function for our proposed optimization model is stated as follows:

$$
\arg \min \sqrt{\frac{1}{m} \sum_{i=1}^{m}\left(a_{i+\tau}-b_{i+\tau}\right)^{2}} ; \quad \forall b \in b_{i}
$$

$$
\text { Subject to } a_{-i-w+\tau} \leq a_{i-w+\tau} \leq \bar{a}_{i-w+\tau}
$$

where $m$ is the total number of data points in the time series. Also, the real and the predicted energy consumption of upcoming phases are shown by $a_{i+\tau}$ and $b_{i+\tau}$ respectively. The solutions' space is defined as $R$ to minimize the fitness function. The optimization problem is to find a global optimum fitness $p^{*}$ and a solution $a^{*} \in \mathbb{R}$ such a way that:

$$
p^{*}=p\left(a^{*}\right) \leq p(a) \quad \forall a \in a_{i}
$$

where $a^{*}$ is the minimum location in the available solutions.

\section{OUR PROPOSED FRAMEWORK}

The infrastructure of our proposed energy consumption prediction model was set up in an experimental room. Fig. 1 shows the installation based WAon the location and function of different elements of the system including the metering infrastructure, data management, and data analytics sections. The metering infrastructure consisted of sensors with bidirectional communication resources to collect and transfer data. The data was recorded in the database management section using NoSQL Database Management System (DBMS) and was transmitted to the data analytics section for analysis and predictions. By integration of data analytics to an optimized hybrid model, the data analytics section created computational models to examine data of electricity consumption concurrently and in real-time. Next, energy consumption patterns of the power lines are generated. In the following sections, we describe the details of the scheme. Fig. 2 depicts the development model.

\section{A. Adaptive update of ARIMA's parameters by ICA}

ICA is a search algorithm based on population, which is based on imperialistic competition and was initially developed by Rajabioun [35]. ICA has been widely adopted for solving optimization problems [36]. The algorithm simulates individuals as countries, which are colonized by other countries based on a cost function to form empires. Countries can be controlled in the ICA algorithm by presenting the social policy of imperialism, and colonies are also subject to some rules for moving toward their relevant imperialists as we describe below. The following equations are defined based on uniform distribution for each moving colony [37]:

$$
\begin{gathered}
x=u(0, \alpha \times d) \\
\theta=u(-\gamma, \gamma)
\end{gathered}
$$

where $x$ is a distance, $\alpha$ is a number higher than $1, d$ is the distance between the colony and the imperialist, and the deviation from the original direction is set by a limit angle $\gamma$. If one empire is lost due to a lack of power, other empires will compete to take its position over colonized countries. Many evolutionary-based algorithms such as GA, Tabu Search (TS), and SA in [37] have been proposed to find a better solution. Nonetheless, evolutionary-based algorithms have limitations because they are considerably slow in finding the optimum solution. With continuous development in this field, a novel evolutionary-based method has been proposed in [38] as the ICA, which can solve difficult optimization problems. ICA not only provides a better solution but it also converges very quickly compared to other ordinary evolutionary-based algorithms [39].

ICA executes the following steps [36]:

1- Randomly generate the primary population for each empire.

2- Move the colonies toward the irrelevant imperialist.

3- Swap the location of a colony and the imperialist if the cost is low.

4- Compute the target function of all empires. 


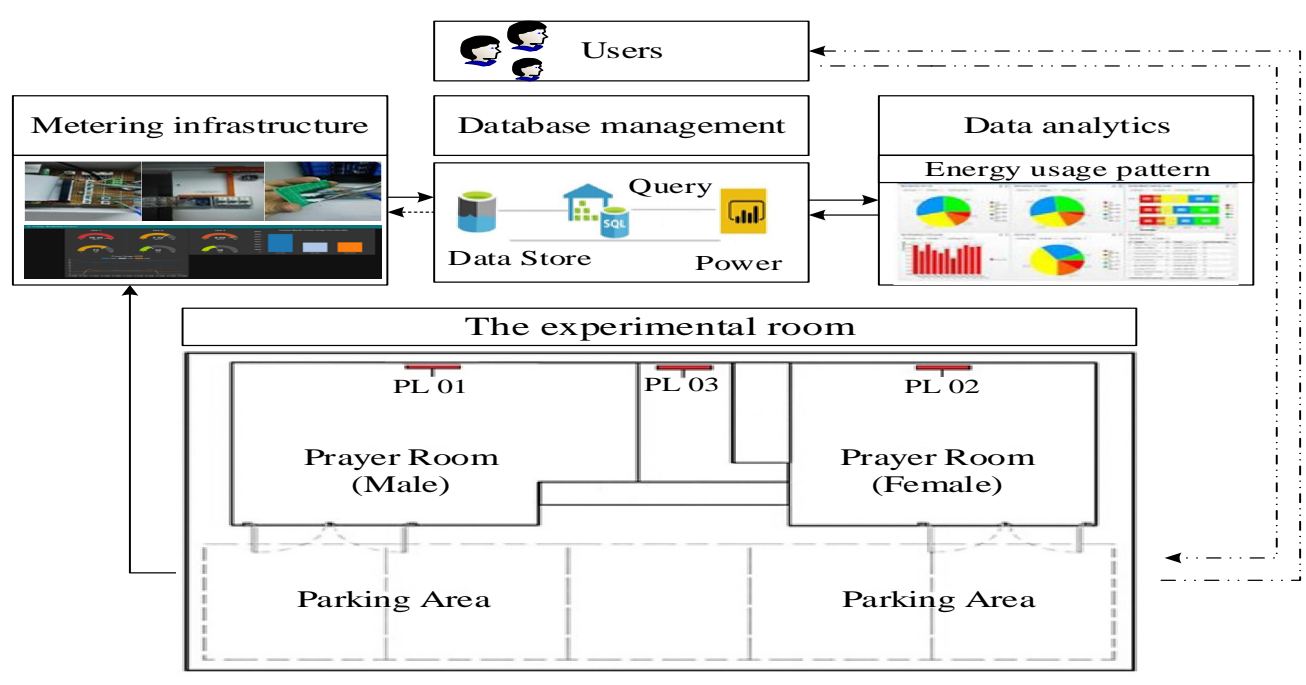

Data flow $\longrightarrow$ Information flow $\rightarrow$.......... Action flow by users $-\cdots+-$

Figure 1: Infrastructure of our proposed model.



Figure 2: Flowchart of our proposed model.

5- Select the feeblest colony and assign it to one of the best empires.

6- Eliminate empires with less power.

7- Stop if only one empire is left. Otherwise, go to step 2.

In this model, the empires of ICA move the average coefficients and the auto-regressive coefficients in the ARIMA model. Let $n$ denote the empire's number and $m$ denote the number of optimized parameters. Therefore, the $i^{t h} \mathrm{em}-$ pire $e_{i}(t)$ in the search space can be defined as $e_{i}(t)=$ $\left(e_{i 1}, e_{i 2}, \ldots, e_{i m}\right)(i=1,2, \ldots, n)$. Also, the $i^{t h}$ empire's movement is denoted as $x_{i}(t)=\left(x_{i 1}, x_{i 2}, \ldots, x_{i m}\right)(i=$ $1,2, \ldots, n)$.

After each movement for each empire $x$, a mutant empire $e_{m u t}^{t+1}$ is generated as follows [37]:

$$
\begin{gathered}
e_{m u t, j}^{t+1}=e_{m 1}^{t}+\operatorname{rand}(.) \times\left(e_{m 2}^{t}-e_{m 3}^{t}\right) \\
e_{m u t, j}=\left(e_{m u t, 1}, e_{m u t, 2}, \cdots, e_{m u t, k}\right)_{1 \times k}
\end{gathered}
$$

The selected empire can be obtained as follows [37]:

$$
e_{n e w, j}^{t+1}=\left(e_{n e w, 1}, e_{n e w, 1}, \cdots, e_{n e w, k}\right)_{1 \times k}
$$

$$
e_{n e w, k}=\left\{\begin{array}{cc}
e_{n e w, k} ; & \text { if rand(.) } \\
e_{k} ; & \text { otherwise }
\end{array}\right.
$$

where $\operatorname{rand}($.$) is a random number between 0$ and 1 . To select the best empire between $e_{\text {move, } k}$ and $e_{n e w, k}$ in order to replace the $i^{t h}$ empire $e_{i}(t)$, the objective function of ICA in this study is described as follows:

$$
e_{j}^{t+1}=\left\{\begin{array}{cc}
e_{\text {move }, j}^{t+1} ; & \text { if } \operatorname{cost}\left(e_{\text {move }, j}^{t+1}\right) \leq \operatorname{cost}\left(e_{\text {new }, j}^{t+1}\right) \\
e_{n e w, j}^{t+1} ; & \text { otherwise }
\end{array}\right.
$$

We performed the experimental analysis by varying the number of empires to guarantee the convergence to a minimum of ICA in different iterations. Based on previous test optimizations [40], we set the iteration limit to 100 .

A control procedure involves regular spreading of residual energy with a population mean $\bar{Z}$, population standard deviation $\sigma_{Z}, Z \sim N\left(\bar{Z}, \sigma_{Z}^{2}\right)$ and $\bar{Z}=\sum_{i=1}^{n} \frac{Z_{i}}{n}$. For any time $t$, the updated procedure of the EWMA method is as follows:

$$
x(i)_{t}=\alpha \bar{Z}_{t}+(1-\alpha) \cdot x(i)_{t-1}
$$

where $\bar{Z}_{t}$ is the residual energy measured at the time, and $x(i)_{t}$ is the forecast for period $t$. The amount $x(i)_{t-1}$ indicates the previous value. On the other hand, $x(0)_{t}$ is the initial value and is set to the previous target or in-control mean $\left(\bar{Z}_{0}\right)$. The average of preliminary data is occasionally used as the initial value of the EWMA, so $x(0)_{t}=\bar{Z}_{0} . \alpha$ is the weight factor known as the smoothing constant and is defined between 0 and 1. This constant, $\alpha$, is a modification element to create a prediction process sensitive to minor or steady drift. This constant defines the depth of memory, and its value varies from between 0 and 1. Since the EWMA-type graphs are used to find minor shifts, $\alpha=0.1$ or 0.2 is often suggested in the literature. The sensitivity of the controlling process is controlled by factor $\alpha$ while defining the rate that affects the prediction process by the current measurement. With an $\alpha$ value of approximately 1 , the prediction involves a small 
memory and is very sensitive to the current variations in the calculated signal. Besides, when the value is approximately 0 , the current data is weighted slightly by the process becoming insensitive to standard nocturnal actions. The smoothing factor [41] that has been recommended is in the interval of 0.05 to 0.25 .

In this work, we analyzed the impact of changing the smoothing constant factor $\alpha$. Table I shows the results obtained where $\alpha$ is $0.095,0.1,0.5$ and 0.9 . If the value of $\alpha$ is equal to 0.095 , the weight of the prior data increased, and the data's dispersion is significantly reduced. If the value of $\alpha$ is equal to or higher than 0.5 , the data's dispersion is reflected immediately. We found that the changing the value of $\alpha$ is very sensitive on EWMA. Therefore, the standard deviation to $\alpha$ has been calculated to quantitatively examine the smoothing effect based on different $\alpha$ for the dataset. If the value of $\alpha$ is decreased, the smoothing effect on the data increases. The experiments demonstrate that the effect of small disruptions is absorbed by $\alpha=0.095$, providing optimum accuracy with a high recognition rate and the lowest number of incorrect alarms. Accordingly, the smoothing factor is set to 0.095 in the examinations. For the control procedure, the mean and the variance of the EWMA statistic are determined from Table I.

$$
\begin{gathered}
M\left(x(i)_{t}\right)=\bar{Z}_{0} \\
V\left(x(i)_{t}\right)=\left(\frac{\sigma_{Z}^{2}}{n}\right)(2-\alpha)(1-\alpha)^{2 t} \theta=u(-\gamma, \gamma)
\end{gathered}
$$

The parameters $\bar{Z}_{0}$ and $\sigma_{Z}^{2}$ are estimated from preliminary samples. The Lower Control Limit $(L C L)$, the Upper Control Limit $(U C L)$, and the Centre Line $(C L)$ of the EWMA chart at time $t$ are determined as follows [21]:

$$
\begin{aligned}
& L C L_{t}=\bar{Z}_{0}-L\left(\frac{\sigma_{Z}}{\sqrt{n}}\right) \sqrt{\left(\frac{\alpha}{(2-\alpha)}\right)(1-\alpha)^{2 t}} \\
& U C L_{t}=\bar{Z}_{0}+L\left(\frac{\sigma_{Z}}{\sqrt{n}}\right) \sqrt{\left(\frac{\alpha}{(2-\alpha)}\right)(1-\alpha)^{2 t}}
\end{aligned}
$$

$L C L_{t}$ and $U C L_{t}$ are calculated based on $t$ because the chart varies with time. If the limits are considered as constant values, then the control limits are changed as follows:

$$
\begin{aligned}
& L C L=\bar{Z}_{0}-L\left(\frac{\sigma_{Z}}{\sqrt{n}}\right) \sqrt{\frac{\alpha}{(2-\alpha)}} \\
& U C L=\bar{Z}_{0}+L\left(\frac{\sigma_{Z}}{\sqrt{n}}\right) \sqrt{\frac{\alpha}{(2-\alpha)}}
\end{aligned}
$$

\section{Performance Evaluation}

Fig. 3 shows the site (prayer room) that was used for the experiment in scenario 1, the power control box including the three power lines (red, yellow and black) and current sensors attached to them. We evaluated the proposed forecasting technique using the data found from a large prayer room fitted with environmental controller actuators comprising numerous lights, fans, and air conditioners (Fig. 3). In scenario 2, we adopted the proposed forecasting technique using the data found from a university library building in the national university of Malaysia. There is a power control box containing the three power lines including lighting devices, an air-conditioning system and computers in the library. The sensors are attached to the power lines in the library building. Different data sets from a library building are collected to verify the forecasting ability of the proposed hybrid model. All data sets are derived from actual building energy usage data.

We divided the prayer room and library building into six parts. The actuators were supplied in each part and six single power lines attached to a core control box. The power lines were attached to the sensor (Fig. 4). A micro-controller (Raspberry $\mathrm{Pi}$ ) is used to read the current (measured in $\mathrm{KW} / \mathrm{h}$ ) and change it to power (Fig. 3). Then, using 4G of memory, the data was transferred to an IBM black mix virtual server and stored into a NoSQL database. This database stores the electricity data collected in real-time manner, IoT devices information, electricity unit price at location, voltage, power, frequency, and power factor. The collected data was then sent to the data analytics section for analysis and prediction. Using the above arrangement, we controlled the power consumption of the prayer room and library building over 24 hours and a 1minute interval frequency. The panel provides real-time power consumption based on $\mathrm{KW} / \mathrm{h}$, power consumption per minutes and total power consumption in a month. Fig. 5 shows the current consumption for all connected power lines. The prediction model can control irrational power consumption by continuous energy monitoring. Table II presents the specifications of the all power lines in each location separately.

The main control box measured the energy data of a set of equipment with the similar input circuit including power consumption, frequency, current power and voltage. The general measurement unit conditions were: accuracy (1\%), voltage $(80-350 \mathrm{~V})$, and current ( $30 \mathrm{~mA}$ to $60 \mathrm{~A})$. Figs. 3 and 4 show the configuration of the microcontroller and Table III presents its specifications.

One of the significant issues in the development of an accurate time series forecasting technique is selecting the input data used to build the prediction model [42]. The parameters of the original ARIMA method were computed and calculated in terms of the Akaike Information Criterion (AIC) [34], and Table IV shows these parameters.

The AIC operator was used to compute the complexity of model. Additionally, the AIC operator was also used in performance tests according to the energy usage results from 3 single power lines. The preceding experimental findings from the simple ARIMA model proposed revealed that the predicting functions must be developed through the loworder diverse equation techniques displayed in Table IV. For explanation, we assume that $x$ is a time series of the ARIMA model [ARMA $(p, q)$ ] involving $n$ cases that can be defined using forecasting equations (24), (25) as follows:

$$
\begin{gathered}
x_{t}=\sum_{i=1}^{p} A_{i} x_{t-1}+\sum_{j=1}^{q} B_{j} \omega_{t-j}+\omega_{t} \\
\hat{x}_{t}=\alpha_{1} x_{t-1}+\cdots+\alpha_{p} x_{t-p}+\omega_{t}=\sum_{i=1}^{p} \alpha_{i} x_{t-i}+\omega_{t}
\end{gathered}
$$


Table I: Level of standard deviation with different smoothing constant factors ( $\alpha$ : Smoothing Constant Factor; $m=$ Standard Deviation; $S(\%)$ : Smoothing

\begin{tabular}{|c|c|c|c|c|c|c|}
\hline & Link1 & & Link2 & & Link3 & \\
\hline$\alpha$ & $m$ & $S(\%)$ & $m$ & $S(\%)$ & $m$ & $S(\%)$ \\
\hline 0.90 & 0.0042 & 10.1 & 0.0041 & 14.12 & 0.0039 & 12.18 \\
\hline 0.70 & 0.0036 & 26.8 & 0.0038 & 23.89 & 0.0041 & 25.84 \\
\hline 0.60 & 0.0034 & 33.8 & 0.0036 & 30.5 & 0.0035 & 34.6 \\
\hline 0.50 & 0.0027 & 38.9 & 0.0025 & 36.9 & 0.0029 & 40.7 \\
\hline 0.40 & 0.0026 & 47.9 & 0.0022 & 45.3 & 0.0028 & 46.5 \\
\hline 0.10 & 0.0013 & 69.9 & 0.0016 & 70.5 & 0.0019 & 73.2 \\
\hline 0.095 & 0.0007 & 85.8 & 0.0009 & 87.6 & 0.0008 & 88.3 \\
\hline
\end{tabular}

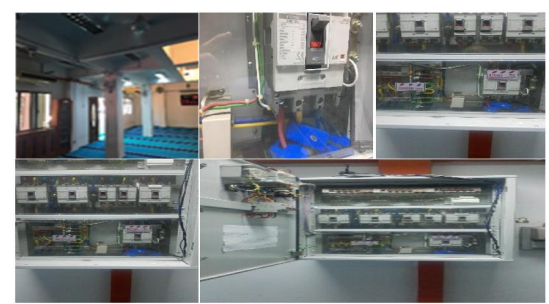

Figure 3: Installation of our energy monitoring system.



Figure 4: Handling and transmission of data utilizing a microcontroller (Raspberry $\mathrm{Pi})$.

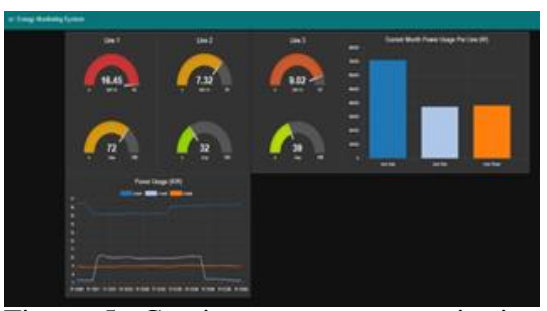

Figure 5: Continuous energy monitoring using our designed system.
Table II: Specifications of the power lines

\begin{tabular}{ll}
\hline \hline Parameter & Value \\
\hline Power consumption & $<1 \mathrm{~V} \mathrm{~A}$ \\
Impedance & $>220 \mathrm{k} \Omega$ \\
Rate & $60 \mathrm{~A}$ \\
Power range & $\mathrm{AC} 100-300 \mathrm{~V} / \mathrm{DC} 5 \mathrm{~V}$ \\
Communication & two-way RS- 485 \\
Voltage rate & $0-370 \mathrm{~V} / \mathrm{AC}$ \\
Frequency & $30-40 \mathrm{~Hz}$ \\
Environment & -25 to $50 \mathrm{C}^{\circ}$ \\
\hline \hline
\end{tabular}

Table III: Specifications of the micro controller

\begin{tabular}{ll}
\hline \hline Item & Value \\
\hline CPU & $32-$ bit ARM-7 $(80 \mathrm{MHz})$ \\
Input power & $110-240 \mathrm{~V}$ AC/15 A/50-60 Hz \\
Maximum current & $15 \mathrm{~A}$ \\
RAM & $16 \mathrm{MB}$ \\
ROM & $8 \mathrm{MB}$ \\
Ethernet L & $10 / 100 \mathrm{Mbps}$ \\
Temperature & $0-60 C^{\circ}$ \\
Port & $\mathrm{RJ}-45$ connector \\
Dimensions & $37 \mathrm{~cm} \times 12 \mathrm{~cm} \times 6 \mathrm{~cm}(\mathrm{~W} \times \mathrm{D} \times$ \\
& $\mathrm{H})$ \\
Weight & $1.50 \mathrm{~kg}$ \\
\hline \hline
\end{tabular}

Table IV: ARIMA model parameters based on points of use

\begin{tabular}{llll}
\hline \hline ARIMA & Line 1 & Line 2 & Line 3 \\
\hline $\mathrm{p}$ & 3 & 5 & 3 \\
$\mathrm{~d}$ & 3 & 1 & 1 \\
$\mathrm{q}$ & 1 & 0 & 0 \\
\hline \hline
\end{tabular}

where $\hat{x}_{t}$ presents the data that is forecasted and $x_{t}$ represents the original results which are obtained. Additionally, unmodified random data with a mean of 0 is indicated by the m-dimensional vector $\omega_{t}$ and the order of the forecast are represented by $\theta=(p, q)$ and covariance matrix of $R$ in which number of auto-regressive expressions and number of lagged prediction errors are defined as $p$ and $q$ respectively. Also, the $m \times m$ coefficient matrices of the MultiVariate (MV) ARIMA model are represented by $B_{1}, \cdots, B_{q}$ and $A_{1}, \cdots, A_{p}$. We have tested the stationary process before implementation of ARIMA based on the MA and AR coefficients as presented in Table V. The optimal values of the MA and AR coefficients is indicated by the data. Notably, the MA and AR coefficients tends to zero as the measurement error increases and as shown in Table V, the MA and AR coefficients are all weak. In fact, the coefficients have to be exactly equal to one. Moreover, the standard deviations of them were between 4.05 and 7.85 which are high and not considerable to be generalized. Also, the data distribution is heterogeneous which was cause low precision. Consequently, the mixed ARIMA is considered the most efficient option. In $\operatorname{ARIMA}(p, d, q), p$ corresponds to the number of auto-regressive terms, $q$ corresponds to the number of lagged forecasting errors and $d$ shows the number of non-seasonal differences. The random errors $\left(\omega_{t}\right)$ have been assumed to be independent with equivalent distribution and a steady variance. The energy usage data of the all power lines could be forecasted using the equations below (Equations 23-25). Fig. 6, Fig. 7, and Fig. 8 illustrate the original ARIMA model, the ICA-enhanced ARIMA and the optimized AIK-EWMA hybrid model, respectively, compared to the prediction results on usage rates for each of the six power lines. To evaluate the model's performance, the main indices such as Average Relative Error (ARE), Root Mean Square Error (RMSE), and Mean Absolute Error (MAE), were measured and demonstrated the prediction accuracy of the 
Table V: AR and MA coefficients of parameters

\begin{tabular}{lll}
\hline \hline ARIMA & Coefficients & $\begin{array}{l}\text { Standard. devia- } \\
\text { tion. }\end{array}$ \\
\hline Line 1 & 1.5031 & 4.0847 \\
AR (1) & 1.4186 & 4.0663 \\
AR (2) & -0.4378 & 5.0778 \\
AR (3) & -0.4131 & 7.0293 \\
MA (1) & 1.7011 & 5.0988 \\
MA (2) & -0.1510 & 5.0728 \\
MA (3) & & \\
Line 2 & -0.7144 & 7.0748 \\
AR (1) & -0.4466 & 4.0677 \\
AR (2) & 1.2873 & 5.0433 \\
AR (3) & 0.4355 & 7.0981 \\
MA (1) & 1.2788 & 5.1067 \\
MA (2) & 0.1314 & 4.0433 \\
MA (3) & & \\
Line 3 & 0.1765 & 4.753 \\
AR (1) & 0.1106 & 4.043 \\
AR (2) & 0.1076 & 7.753 \\
AR (3) & -0.3081 & 6.233 \\
MA (1) & 0.2944 & 4.053 \\
MA (2) & 0.8733 & 5.012 \\
MA (3)
\end{tabular}

models. The indices used include [22]:

$$
\begin{gathered}
R M S E=\sqrt{\sum_{i=1}^{n} \frac{\left(y_{i}-\hat{y}_{i}\right)^{2}}{n}} \\
M A E=\frac{\sum_{i=1}^{n}\left|y_{i}-\hat{y}_{i}\right|}{n} \\
A R E=\frac{\sum_{i=1}^{n}\left|\hat{y}_{i}-y_{i}\right| / y_{i}}{n}
\end{gathered}
$$

where $y_{i}$ is the real data, and $\hat{y}_{i}$ is the predicted value of $y_{i}$. Tables VI to VIII show the performance of each prediction model according to the forecasted consumption of the six power lines in the prayer room and library building compared to their forecasting data. Table VI presents the power usage predicted data of the ARIMA model, and Fig. 6 shows that the ARIMA model could explain the diversity and change of the time series. The elementary ARIMA model can describe the energy consumption variation with acceptable performance. However, the low precision of ARIMA prediction could not satisfy the demand for energy storage in actual electricity markets. The present study also developed an improved version of ARIMA method, namely the ARIMA-EWMA method. The optimal discount factor of the EWMA controller was determined based on Tseng's method [43]. This factor was obtained by minimizing the mean square error of the process output at each run. The optimal variable discount factor was derived and applied to the general ARIMA processes. The results of the ARIMA-EWMA model show that the proposed model performed better than the conventional EWMA controller in minimizing the process variation when there is primary setup bias.

To effectively forecast the energy usage, we proposed ICA to increase the parameters of the ARIMA technique. For all power lines, the ICA method was applied for parameter optimization in the ARIMA method. Fig. 6 shows the actual
Table VI: Indices of ARIMA model

\begin{tabular}{lllll}
\hline \hline Scenario & & Line 1 & Line 2 & Line 3 \\
\hline \multirow{3}{*}{1} & ARE & 0.42 & 0.4 & 0.29 \\
& MAE & 1.1 & 0.8 & 0.74 \\
& RMSE & 0.9 & 1.8 & 0.75 \\
\hline \multirow{2}{*}{2} & ARE & 0.39 & 0.36 & 0.23 \\
& MAE & 1.12 & 1.1 & 0.8 \\
& RMSE & 0.84 & 1.3 & 0.95 \\
\hline \hline
\end{tabular}

Table VII: Incidence of the ARIMA-EWMA model

\begin{tabular}{lllll}
\hline \hline Scenario & & Line 1 & Line 2 & Line 3 \\
\hline \multirow{3}{*}{1} & ARE & 0.36 & 0.3 & 0.25 \\
& MAE & 0.53 & 1.05 & 0.67 \\
& RMSE & 0.77 & 0.91 & 0.68 \\
\hline \multirow{2}{*}{2} & ARE & 0.33 & 0.23 & 0.20 \\
& MAE & 0.48 & 0.95 & 0.53 \\
& RMSE & 0.85 & 1.20 & 0.94 \\
\hline \hline
\end{tabular}

Table VIII: Incidence of the ICA-optimized ARIMA model

\begin{tabular}{lllll}
\hline \hline Scenario & & Line 1 & Line 2 & Line 3 \\
\hline \multirow{3}{*}{1} & ARE & 0.4 & 0.3 & 0.27 \\
& MAE & 0.8 & 0.45 & 0.32 \\
& RMSE & 0.9 & 1.2 & 0.72 \\
\hline \multirow{2}{*}{2} & ARE & 0.35 & 0.28 & 0.23 \\
& MAE & 0.58 & 0.35 & 0.25 \\
& RMSE & 1.9 & 1.5 & 1.1 \\
\hline \hline
\end{tabular}

and predicted results. The changes in the time series were better described by the ICA optimized ARIMA method. As Fig. 6 and Fig. 7 show, all models presented the same modes as noted in real data. Nevertheless, there are great variations were noted among the data predicted from the original ARIMA method and the actual data. Table VIII shows the assessment indicators of the ICA enhanced ARIMA.

The key findings of the designed algorithm reveal a hybrid between the ARIMA model and the ICA can increase the accuracy of prediction. We obtain a considerable improvement for energy usage prediction in the large prayer room and library building as the optimized AIK-EWMA hybrid reduces the predicted error because of the ICA-enhanced ARIMA technique for all power lines in each location separately. Table IX presents the list of indicators. Fig. 8 presents the latest predicted results. These results show an enhancement of the AIK-EWMA hybrid model in comparison with the basic ARIMA and ICA-enhanced ARIMA models. According to the measures defined above, the indices of diverse models for the all power lines in each location demonstrate the performance of our proposed method. Overall, the power usage indicators and forecasted data improved with the novel AIK-EWMA hybrid enhanced model. For instance, regarding the ARE in line 1, the values range between $42 \%$ and $40 \%$ for the original ARIMA process and the ICA-optimized ARIMA technique. However, improved AIK-EWMA hybrid method reduced the ARE to $33 \%$. In the additional instance of RMSE in line 2, the value of the original ARIMA technique was 1.8575 , and the RMSE of the ICA enhanced ARIMA method was reduced to 1.2. In contrast, the RMSE of the enhanced AIK-EWMA hybrid algorithm was 1.02 . The experiments from dissimilar power lines consistently show similar outcomes, demonstrating that the optimized AIK-EWMA hybrid model has better 


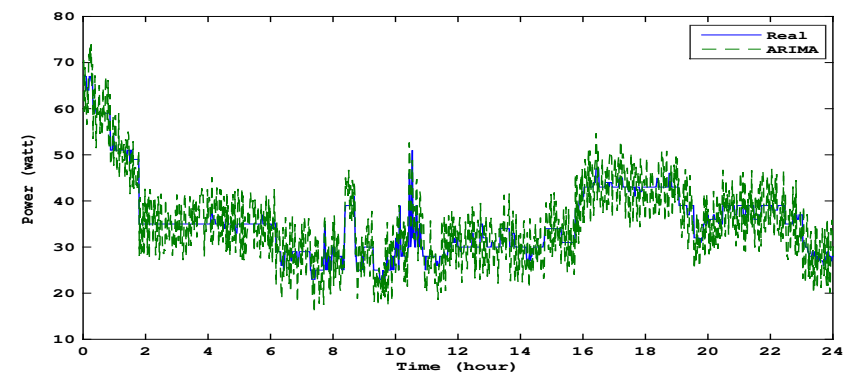

(a) Scenario 1:Line 1

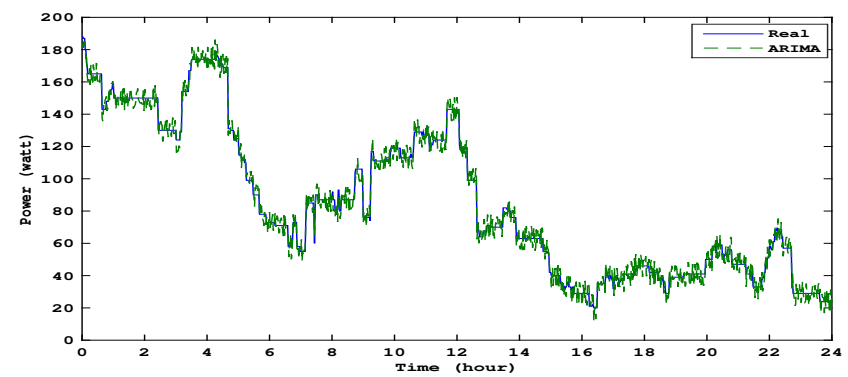

(b) Scenario 1:Line 2



(c) Scenario 1:Line 3

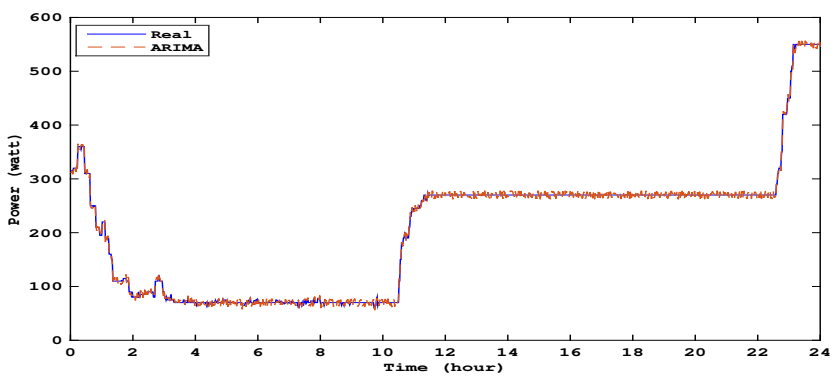

(d) Scenario 2:Line 1

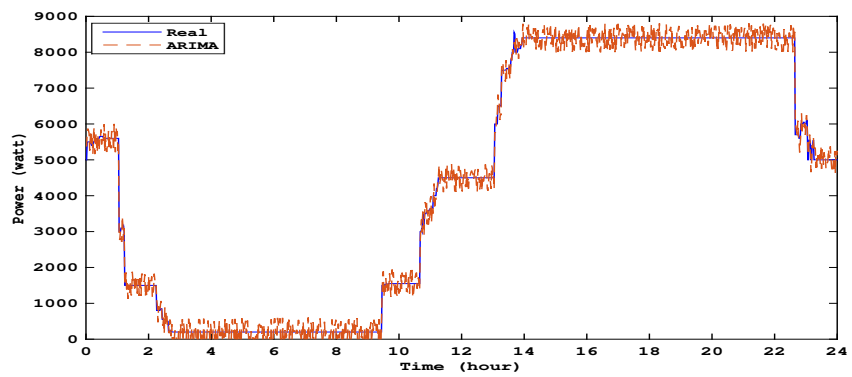

(e) Scenario 2:Line 2



(f) Scenario 2:Line 3

Figure 6: Actual and predicted results of the ARIMA model.

performance than the ICA-enhanced ARIMA method, which is superior to the original ARIMA method. Additionally, the results of AIK-EWMA hybrid model show that the proposed model performs better than the ARIMA-EWMA model. For instance, RMSE in line 2 had a value of 0.91 for the ARIMAEWMA model, and a lower value of 1.09 for the AIK-EWMA model. Regarding the ARE results in line 3, the values are 0.25 and 0.21 for the ARIMA-EWMA model and the AIKEWMA hybrid model respectively. Table VII and Table IX show the results of these models wherein the AIK-EWMA hybrid model achieves the lowest RMSE, MAE, and ARE as assessed for each of the six power lines. This result demonstrates the potential of the optimized AIK-EWMA to enhance the precision of the forecasted values as compared to the other two predicted trends. By considering various proposed models in this research, we have demonstrated the outcomes with high accuracy using superior non-linear and linear techniques; in particular, when both ICA-optimised ARIMA and AIK-EWMA hybrid model models show accurate and strong prediction robustness. Because of the acceptable capability of the ARIMA model in estimation of the linear part of data, we have demonstrated that using the ICA algorithm can efficiently improve the predicted results for the non-linear parts of the model.
Table IX: Incidence of the AIK-EWMA model

\begin{tabular}{lllll}
\hline \hline Scenario & & Line 1 & Line 2 & Line 3 \\
\hline \multirow{2}{*}{1} & ARE & 0.33 & 0.24 & 0.21 \\
& MAE & 0.71 & 0.28 & 0.24 \\
& RMSE & 0.63 & 1.09 & 0.61 \\
\hline \multirow{2}{*}{2} & ARE & 0.29 & 0.20 & 0.18 \\
& MAE & 0.65 & 0.18 & 0.15 \\
& RMSE & 0.96 & 1.10 & 0.95 \\
\hline \hline
\end{tabular}

Fig. 9 shows the EWMA control chart, and the area where a failure occurs is represented by the shaded area in the control chart. According to the chart, one of the two failures was detected. The model predicted the failures (as denoted by a square dot) by approximately 10-time units before the actual failures occurred.

In the next step, we compare the overall prediction performance of our algorithm selection method compared to using a single prediction algorithm for all the houses. By applying the time series prediction algorithm suggested by the RF method for a given house, we obtain 1.909 MSE averaged over all houses, where this average MSE value is 1.828 if we were to use an oracle (the case where we know all the best prediction algorithms for each house ahead of time). This results in only $4.43 \%$ additional error on top of the oracle. However, 


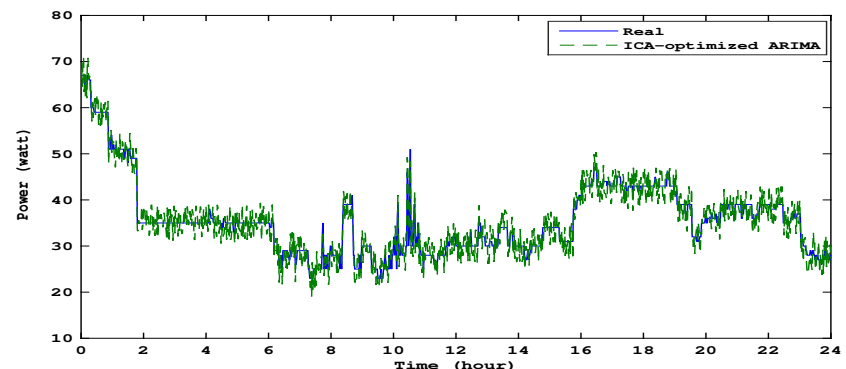

(a) Scenario 1:Line 1

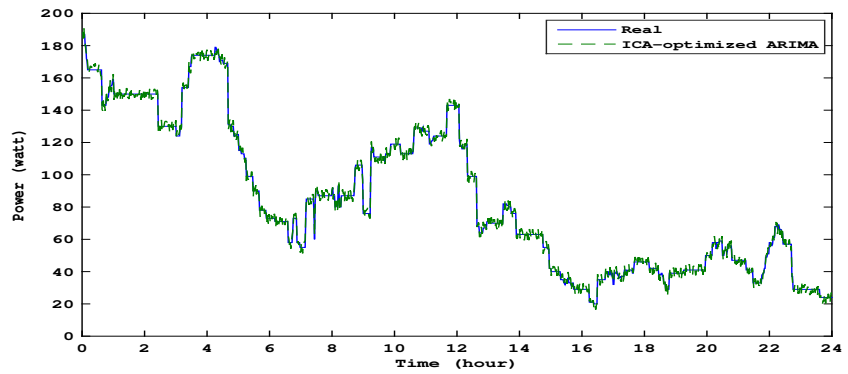

(b) Scenario 1:Line 2

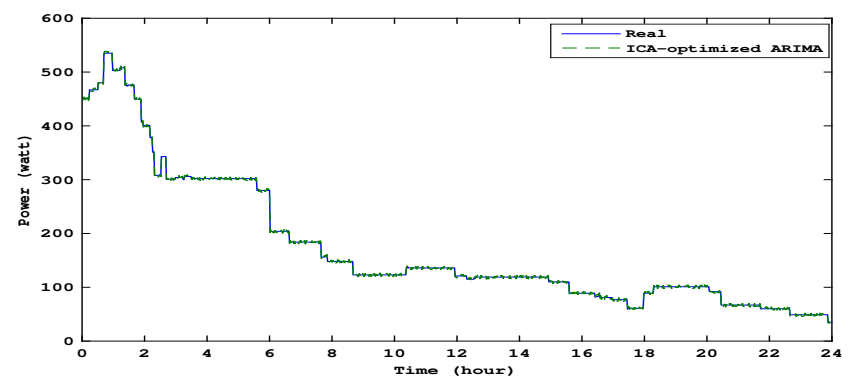

(c) Scenario 1:Line 3

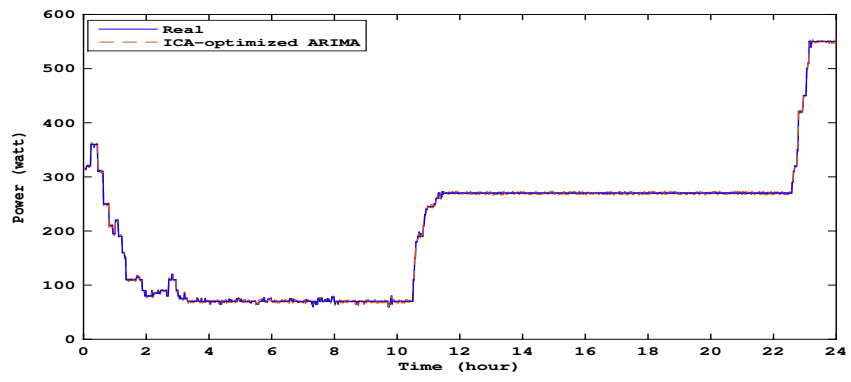

(d) Scenario 2:Line 1



(e) Scenario 2:Line 2



(f) Scenario 2:Line 3

Figure 7: Actual and predicted results of the ICA-optimized ARIMA model.

Table X: Incidence of the ARIMA model enhanced by ICA

\begin{tabular}{lllll}
\hline \hline Scenario & MODEL & (ARIMA/AIK- & $\begin{array}{l}\text { (ARIMA/ICA- } \\
\text { optimized ARIMA) }\end{array}$ & $\begin{array}{l}\text { (ICA-optimized } \\
\text { ARIMA/AIK- } \\
\text { EWMA) }\end{array}$ \\
& & EWMA) & Line 2 & Line 3 \\
\hline 1 & Power line & Line 1 & Rejected & Accepted \\
& Sign Test & Accepted & Accepted & Accepted \\
\hline 2 & Wilcoxon's s-r Test & Rejected & Rejected & Accepted \\
& Sign Test & Rejected & Accepted & Accepted \\
\hline \hline
\end{tabular}

if we use a single prediction algorithm, the average MSE values are as follows: 2.214 for TESLA, 2.246 for ARIMA, 3.296 for LSTM, 4.16 for Holt-Winters. This corresponds to $21.1 \%$ additional error in the best case (using TESLA), and up to $127.5 \%$ additional error. All of our experimental results verify that an algorithm selection-based method, to find the best prediction algorithm for the energy consumption of individual houses, is crucial to improve the overall prediction performance.

\section{Prediction accuracy testing}

Predictive accuracy testing is vital when assessing the stability of prediction models based on the distribution of prediction error in each model. In many applications, the forecast error function is defined as $g\left(y_{t}, \hat{y}_{i t}\right)=g\left(e_{i t}\right)$. The $\left[g\left(e_{i t}\right)-g\left(e_{j t}\right)\right]$ is the difference between the two forecast error functions. The null hypothesis of equal forecast accuracy that used for the two forecast error functions is $E\left[g\left(e_{i t}\right)\right]=E\left[g\left(e_{j t}\right)\right]$, which means that $d_{t}=0$. The loss differential is represented by $d_{t}$, whereas $d_{t} \equiv\left[g\left(e_{i t}\right)-g\left(e_{j t}\right)\right]$. Therefore, the equal precision null hypothesis corresponds to the null assumption that the population means of the loss differential series is 0 .

\section{A. Sign test}

The null hypothesis includes a 0 median loss differential: $\operatorname{med}\left(g\left(e_{i t}\right)-g\left(e_{j t}\right)\right)=0$. If $i i d$ is the loss differential series, the number of mean differential values in a sample of size $T$ include the binomial distribution with parameters $T$ and $\frac{1}{2}$ under the null assumption. Therefore, the statistical test is as 


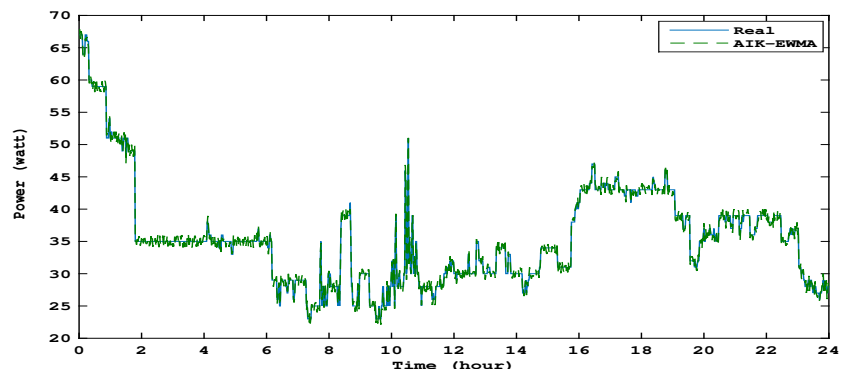

(a) Scenario 1:Line 1



(b) Scenario 1:Line 2

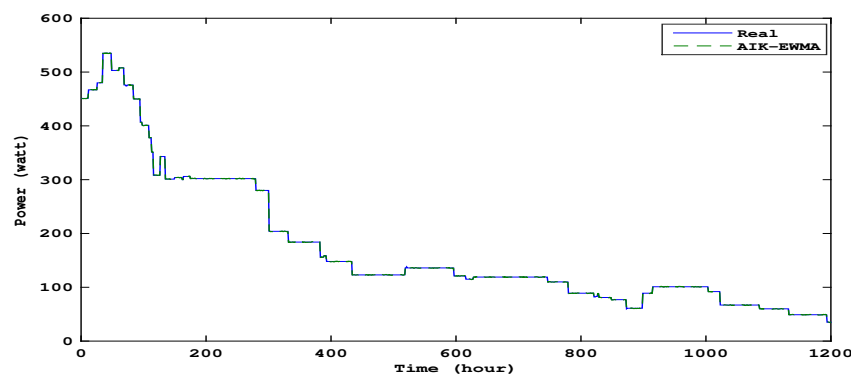

(c) Scenario 1:Line 3

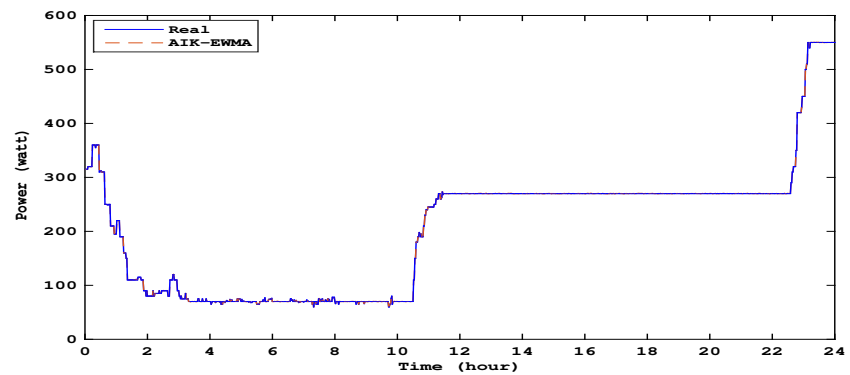

(d) Scenario 2:Line 1

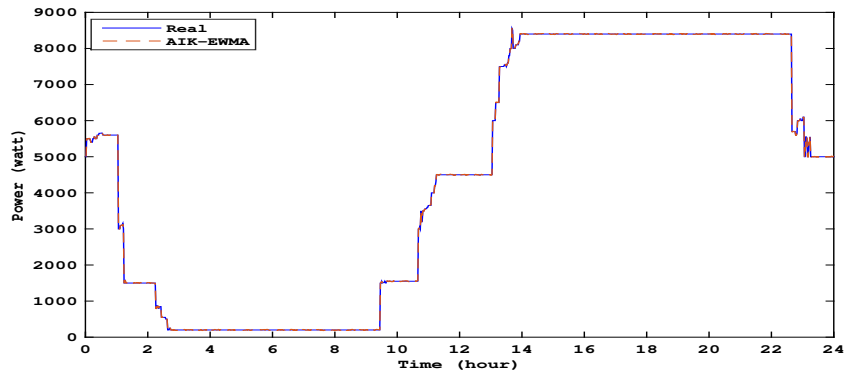

(e) Scenario 2:Line 2

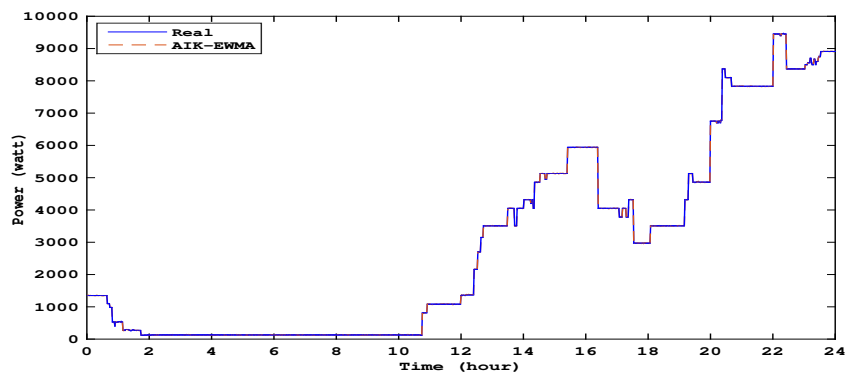

(f) Scenario 2:Line 3

Figure 8: Predicted results of the optimized hybrid model.

follows:

$$
S=\sum_{t=1}^{T} I+\left(d_{t}\right)
$$

where $I+\left(d_{t}\right)=\left\{\begin{array}{ll}1 ; & \text { if } d_{t}>0 \\ 0 ; & \text { otherwise }\end{array}\right.$ We evaluated the significance using a cumulative binomial distribution table. In the examples, the sign statistical test is the standard norm:

$$
S=\frac{S-0.5 T}{\sqrt{0.25 T}} \sim N(0,1)
$$

\section{B. Wilcoxon's signed-rank test}

This test is more effective than the sign test. This test has a distribution-free process requiring differential loss symmetry. The statistical test is as follows:

$$
S=\sum_{t=1}^{T} I+\left(d_{t}\right) \operatorname{Rank}\left(\left|d_{t}\right|\right)
$$

We report the sum of the positive absolute values. In a precise limited sample, critical values of statistical testing are invariant to the loss differential distribution and have been arranged as zero-mean or symmetric. The regular norm is reported as follows:

$$
S=\frac{S-\frac{T(T+1)}{4}}{\sqrt{\frac{T(T+1)(2 T+1)}{24}}} \sim N(0,1)
$$

For the all power lines, we examined each prediction technique jointly with every other method. Based on the comparison of the results shown in Table X, some of the hypothesis tests have been excluded because of the considerable variations among the three methods. For instance, we obtained a rejection by the sign test in the ARIMA and ICA-optimized ARIMA in the second power line; however, we obtained an acceptance with the Wilcoxon's signed test. There are differences existed between two models. Nevertheless, the response was accepted in some areas, indicating the inability of the tests to effectively distinguish among the forecasted results. In this section, we have discussed the comparative result of the normalized error values of power prediction between the related works and our ICA-optimized ARIMA model (Table XI shows a stacking model [26] and a hybrid nature-inspired optimization algorithm [22]). We used the normalized error of power [44] to measure the error between the predicted and actual power data. The measure calculated the deviation of the actual power data $y_{i}$ to the estimated power value $\hat{y}_{i}$ over the specified period 


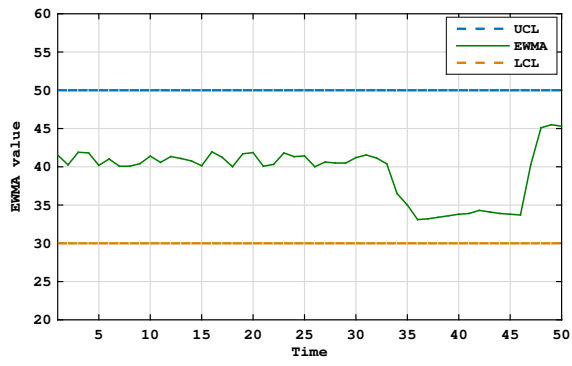

(a) Line 1

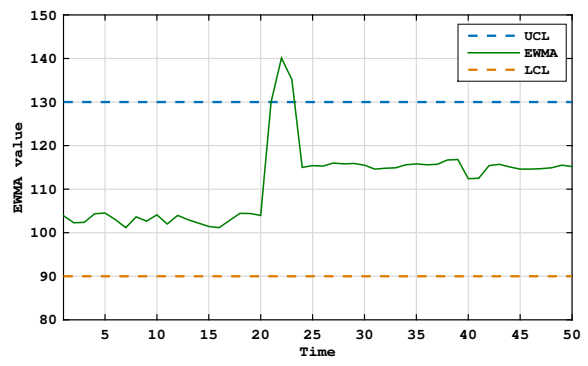

(b) Line 2

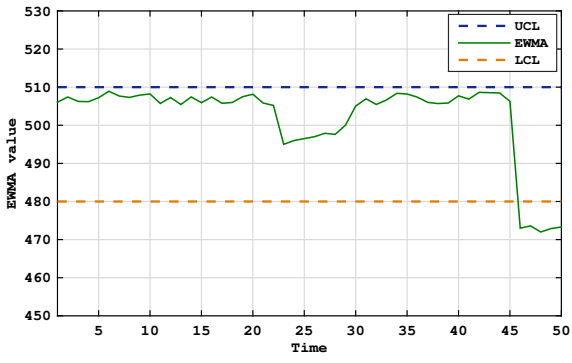

(c) Line 3

Figure 9: EWMA control chart.

$(T)$ in which the total energy consumption was taken. The percentage normalized error of power is expressed by (33):

$$
\text { Normalized error of Power }=\frac{\sum_{\mathrm{T}}\left|y_{i}-\hat{y}_{i}\right|}{y_{i}} \times 100
$$

Since the proposed approach is an optimization-based technique for energy consumption prediction using the ICAoptimized ARIMA and the EWMA, the computational complexity of this technique depends on several operators, that affect computing time, including time input data in the ARIMA, number of generations in the ICA, population size in the ICA, etc. These factors can create an NP computational time problem in the approach.

Calculation of computation Time (CT) is especially necessary in the real-time applications when the prediction algorithm should quickly predict the power consumption. In this study, the stopwatch timer functions, tic and toc, are used to calculate the computation time. Invoking tic starts the timer, and the next toc reads the elapsed time in MATLAB. The CPU time returns the total CPU time (in seconds). The line graph shows the comparison of the average of computation time on the ARIMA-EWMA, ICA-optimized ARIMA and AIK-EWMA models for 30 runs. When comparing the data resulting from the plot, the average time needed for ICAoptimized ARIMA calculation is approximately 0.6 (s). The ARIMA-EWMA has high computation time which is about 0.85 (s). In contrast, AIK-EWMA model has the lowest computation time of 0.43 (s).

As we can see, ARIMA-EWMA dramatically lowered the computation time. In other words, it was evident that the computation time of the proposed ICA-optimized ARIMA was lower when using ICA rules in the integration of the new data set than ARIMA-EWMA by optimizing ARIMA parameters after employing basic ARIMA model. Fig. 10 shows the changes in the computation time for the ARIMAEWMA, ICA-optimized ARIMA and AIK-EWMA methods. The computational time taken for energy consumption prediction is reduced to half the time in comparison with that of the methods described in the literature.

\section{CONCLUSION}

In this work, we have proposed a novel optimized AIKEWMA hybrid prediction model based on ICA-optimized ARIMA to predict the precise power usage of actuation units

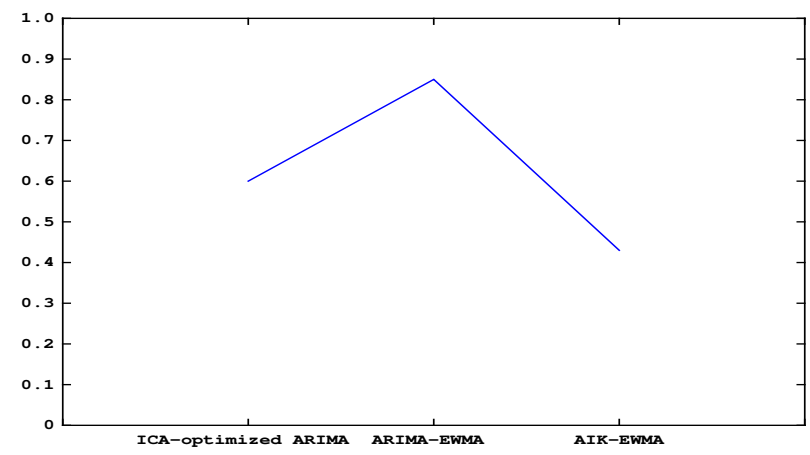

Figure 10: Average of computation time.

for green building environments. We evaluated the performance of the enhanced AIK-EWMA hybrid model based on the power usage results of actuator tools from six areas which independently control the setting of a large multi-purpose prayer room and library building. The results obtained show that, based on the forecasting and prediction capabilities of the innovative AIK-EWMA hybrid method, the existing nonoptimized ARIMA model can be created. Additionally, the results obtained also show that the ICA-optimized ARIMA is a promising technique for planning and enhancing the ARIMA model in energy usage prediction for large-scale buildings. The novel AIK-EWMA hybrid predictive modeling method provides numerous advantages. First, the use of the ICA method addresses the shortcomings of the ARIMA that is incapable to change the structure of the model whenever the time series data encounters novel data. Also, the model enhanced by ICA makes possible the optimal modification of modeling elements to cope with fluctuating time series results utilized in forecasting energy usage. An optimized AIK-EWMA hybrid model, developed by merging ICA with ARIMA, can adequately predict accuracy as demonstrated by the results obtained in terms of monitoring diverse arrangements of building control tools driven by six various power lines. We have also shown that this work outperforms the models used in our comparisons. The proposed model provided better predictive performance by normalized error values, ranging from $3 \%-47 \%$, which outperform the models used in our performance comparison. Furthermore, the proposed method is comparatively simple to execute and can be deployed in various residential and functional areas. Generally, 
Table XI: Comparison of the normalized error of power between the AIK-EWMA model and previous works.

\begin{tabular}{lllll}
\hline \hline & & \multicolumn{2}{c}{ \% normalized error of power } & \\
\hline Scenario & MODEL & Stacking model [26] & Hybrid algorithm [22] & Proposed model \\
\hline \multirow{3}{*}{1} & Line 1 & 57.6 & 35.3 & 18.2 \\
& Line 2 & 76.5 & 61.3 & 47.2 \\
& Line 3 & 22.3 & 18.6 & 3.7 \\
\hline \multirow{2}{*}{2} & Line 1 & 65.3 & 48.7 & 30.5 \\
& Line 2 & 82.5 & 73.3 & 52.8 \\
& Line 3 & 31.3 & 22.5 & 5.6 \\
\hline \hline
\end{tabular}

the proposed technique can be particularly advantageous as a precise instrument to estimate the extensive energy usage of large-scale buildings thereby playing a vital role in global energy consumption issues. This work aims to restructure power administration, control, and estimation into one single system. So, the ineffective power usage can be minimized, thereby reducing increasing power expenses. Running such a system can result in different advantages and benefits; the most obvious benefit is the significant decrease in the cost of energy consumption. Furthermore, the mobile feature of our proposed implementation which uses low-cost Raspberry Pi's hardware as the central architecture makes our solution highly scalable and cost-effective. The limitation part of this study is that there are some other factors such as temporal granularity, scale, energy type and building type that can affect the prediction outputs at the same time.

\section{ACKNOWLEDGMENT}

The authors wish to thank the Ministry of Higher Education Malaysia and Universiti Kebangsaan Malaysia (grant ID: GGPM-2020-029 and grant ID: PP-FTSM-2020) for supporting and funding this work.

\section{REFERENCES}

[1] U. EIA, "Energy information administration "international energy outlook"," 2010.

[2] O. IEA, "Energy and climate change, world energy outlook special report," 2015.

[3] C. Yin, S. Dadras, X. Huang, Y. Chen, and S. Zhong, "Optimizing energy consumption for lighting control system via multivariate extremum seeking control with diminishing dither signal," IEEE Transactions on Automation Science and Engineering, vol. 16, no. 4, pp. 1848-1859, 2019.

[4] S. Li, K. Deng, and M. Zhou, "Sensitivity analysis for building energy simulation model calibration via algorithmic differentiation," IEEE Transactions on Automation Science and Engineering, vol. 14, no. 2, pp. 905-914, 2016.

[5] H. Wang and P. Mancarella, "Towards sustainable urban energy systems: high resolution modelling of electricity and heat demand profiles," in 2016 IEEE International Conference on Power System Technology (POWERCON). IEEE, 2016, pp. 1-6.

[6] T. Weng and Y. Agarwal, "From buildings to smart buildings—-sensing and actuation to improve energy efficiency," IEEE Design \& Test of Computers, vol. 29, no. 4, pp. 36-44, 2012.

[7] B. Hammi, R. Khatoun, S. Zeadally, A. Fayad, and L. Khoukhi, "IoT technologies for smart cities," IET Networks, vol. 7, no. 1, pp. 1-13, 2017.

[8] F. Tao, Y. Feng, L. Zhang, and T. W. Liao, "Clps-ga: A case library and pareto solution-based hybrid genetic algorithm for energy-aware cloud service scheduling," Applied Soft Computing, vol. 19, pp. 264 279, 2014

[9] S. Zeadally, S. U. Khan, and N. Chilamkurti, "Energy-efficient networking: past, present, and future," The Journal of Supercomputing, vol. 62, no. 3, pp. 1093-1118, 2012.
[10] A. Farayez, M. B. I. Reaz, and N. Arsad, "Spade: activity prediction in smart homes using prefix tree based context generation," IEEE Access, vol. 7, pp. 5492-5501, 2018.

[11] M. R. Alam, M. B. I. Reaz, and M. M. Ali, "Speed: An inhabitant activity prediction algorithm for smart homes," IEEE Transactions on Systems, Man, and Cybernetics-Part A: Systems and Humans, vol. 42, no. 4, pp. 985-990, 2011.

[12] K. Sopian, A. Zaharim, S. Mat, A. Fazlizan, N. Maseran, M. A. M. Safari, A. Jedi, and A. A. Rahim, "The theoretical framework of smart energy management system for rural area in mersing malaysia," in 2017 International Conference in Energy and Sustainability in Small Developing Economies (ES2DE). IEEE, 2017, pp. 1-5.

[13] C. Deb, F. Zhang, J. Yang, S. E. Lee, and K. W. Shah, "a review on time series forecasting techniques for building energy consumption," Renewable and Sustainable Energy Reviews, vol. 74, pp. 902-924, 2017.

[14] S. I. Alzahrani, I. A. Aljamaan, and E. A. Al-Fakih, "Forecasting the spread of the covid-19 pandemic in saudi arabia using arima prediction model under current public health interventions," Journal of infection and public health, vol. 13, no. 7, pp. 914-919, 2020.

[15] S. Shahriari, M. Ghasri, S. Sisson, and T. Rashidi, "Ensemble of arima: combining parametric and bootstrapping technique for traffic flow prediction," Transportmetrica A: Transport Science, vol. 16, no. 3, pp. $1552-1573,2020$.

[16] M. R. Alam, M. B. I. Reaz, and M. A. M. Ali, "A review of smart homes-past, present, and future," IEEE transactions on systems, man, and cybernetics, part $C$ (applications and reviews), vol. 42, no. 6, pp. 1190-1203, 2012.

[17] T. Van Calster, B. Baesens, and W. Lemahieu, "profarima: a profit-driven order identification algorithm for arima models in sales forecasting," Applied Soft Computing, vol. 60, pp. 775-785, 2017.

[18] E. M. de Oliveira and F. L. C. Oliveira, "forecasting mid-long term electric energy consumption through bagging arima and exponential smoothing methods," Energy, vol. 144, pp. 776-788, 2018.

[19] J. Geng, M.-L. Huang, M.-W. Li, and W.-C. Hong, "hybridization of seasonal chaotic cloud simulated annealing algorithm in a svr-based load forecasting model," Neurocomputing, vol. 151, pp. 1362-1373, 2015.

[20] E. Abdollahi and R. Lahdelma, "Decomposition method for optimizing long-term multi-area energy production with heat and power storages," Applied Energy, vol. 260, p. 114332, 2020.

[21] V. Alevizakos, K. Chatterjee, and C. Koukouvinos, "The triple exponentially weighted moving average control chart," Quality Technology \& Quantitative Management, pp. 1-29, 2020.

[22] S. Goudarzi, M. H. Anisi, N. Kama, F. Doctor, S. A. Soleymani, and A. K. Sangaiah, "Predictive modelling of building energy consumption based on a hybrid nature-inspired optimization algorithm," Energy and Buildings, vol. 196, pp. 83-93, 2019.

[23] H. A. Le Thi, X. T. Vo, and T. P. Dinh, "Feature selection for linear SVMs under uncertain data: Robust optimization based on difference of convex functions algorithms," Neural Networks, vol. 59, pp. 36-50, 2014.

[24] G. E. Box, G. M. Jenkins, G. C. Reinsel, and G. M. Ljung, Time series analysis: forecasting and control. John Wiley \& Sons, 2015.

[25] J. Kennedy et al., "Encyclopedia of machine learning," Particle swarm optimization, pp. 760-766, 2010.

[26] R. Wang, S. Lu, and W. Feng, "a novel improved model for building energy consumption prediction based on model integration," Applied Energy, vol. 262, p. 114561, 2020.

[27] L. Suganthi and A. A. Samuel, "Energy models for demand forecasting-a review," Renewable and sustainable energy reviews, vol. 16, no. 2, pp. 1223-1240, 2012.

[28] H. Shareef, M. S. Ahmed, A. Mohamed, and E. Al Hassan, "Review on home energy management system considering demand responses, smart technologies, and intelligent controllers," Ieee Access, vol. 6, pp. 24 498-24 509, 2018. 
[29] Y. Kim, H.-g. Son, and S. Kim, "Short term electricity load forecasting for institutional buildings," Energy Reports, vol. 5, pp. 1270-1280, 2019.

[30] S. Barak and S. S. Sadegh, "Forecasting energy consumption using ensemble arima-anfis hybrid algorithm," International Journal of Electrical Power \& Energy Systems, vol. 82, pp. 92-104, 2016.

[31] P. Sen, M. Roy, and P. Pal, "Application of arima for forecasting energy consumption and ghg emission: A case study of an Indian pig iron manufacturing organization," Energy, vol. 116, pp. 1031-1038, 2016.

[32] $\mathrm{C}$. $\mathrm{Li}$ and J.-W. Hu, "A new arima-based neuro-fuzzy approach and swarm intelligence for time series forecasting," Engineering Applications of Artificial Intelligence, vol. 25, no. 2, pp. 295-308, 2012.

[33] Z. Zhou, F. Xiong, B. Huang, C. Xu, R. Jiao, B. Liao, Z. Yin, and J. Li, "Game-theoretical energy management for energy internet with big data-based renewable power forecasting," IEEE Access, vol. 5, pp. 5731-5746, 2017.

[34] S. Goudarzi, M. N. Kama, M. H. Anisi, S. A. Soleymani, and F. Doctor "Self-organizing traffic flow prediction with an optimized deep belief network for internet of vehicles," Sensors, vol. 18, no. 10, p. 3459, 2018.

[35] R. Rajabioun, F. Hashemzadeh, E. Atashpaz-Gargari, B. Mesgari, and F. R. Salmasi, "Decentralized pid controller design for a MIMO evaporator based on colonial competitive algorithm," IFAC Proceedings Volumes, vol. 41, no. 2, pp. 9952-9957, 2008.

[36] M. Sharifi and H. Mojallali, "Design of iir digital filter using modified chaotic orthogonal imperialist competitive algorithm," pp. 1-6, 2013.

[37] E. Atashpaz-Gargari and C. Lucas, "Imperialist competitive algorithm: an algorithm for optimization inspired by imperialistic competition," pp. 4661-4667, 2007.

[38] S. Hosseini and A. Al Khaled, "A survey on the imperialist competitive algorithm metaheuristic: implementation in engineering domain and directions for future research," Applied Soft Computing, vol. 24, pp. 1078-1094, 2014.

[39] Z. Zhou, F. Xiong, B. Huang, C. Xu, R. Jiao, B. Liao, Z. Yin, and J. Li, "Game-theoretical energy management for energy internet with big data-based renewable power forecasting," IEEE Access, vol. 5, pp. 5731-5746, 2017.

[40] N. Kumar, B. Tyagi, and V. Kumar, "multiarea deregulated automatic generation control scheme of power system using imperialist competitive algorithm based robust controller," IETE journal of Research, vol. 64, no. 4, pp. 528-537, 2018.

[41] P. Cisar, S. Bošnjak, and S. M. Cisar, "ewma algorithm in network practice," International Journal of Computers Communications \& Control, vol. 5, no. 2, pp. 160-170, 2010.

[42] S. Amid and T. Mesri Gundoshmian, "Prediction of output energies for broiler production using linear regression, ann (mlp, rbf), and anfis models," Environmental Progress \& Sustainable Energy, vol. 36, no. 2, pp. 577-585, 2017.

[43] S.-T. Tseng, H.-C. Mi, and I.-C. Lee, "A multivariate ewma controller for linear dynamic processes," Technometrics, vol. 58, no. 1, pp. 104-115, 2016.

[44] N. Batra, J. Kelly, O. Parson, H. Dutta, W. Knottenbelt, A. Rogers, A. Singh, and M. Srivastava, "Nilmtk: an open source toolkit for non-intrusive load monitoring," in Proceedings of the 5th international conference on Future energy systems, 2014, pp. 265-276.

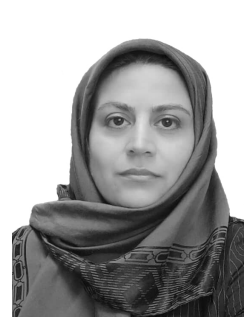

Shidrokh Goudarzi received her Ph.D. degree in communication system and wireless network from Malaysia-Japan International Institute of Technology (MJIIT), Universiti Teknologi Malaysia (UTM). In 2014, She received three-year full scholarship to study Ph.D. at (UTM). Then, She joined the Department of Advanced Informatics School at Universiti Teknologi Malaysia as a Postdoctoral Fellow from 2018 to 2019. Currently, she is a senior lecturer at National University of Malaysia. She also serves as reviewer for many journals such as IEEE Transactions on Industrial Informatics, IEEE Transactions on Artificial Intelligence, IEEE Systems and IEEE Access. Her research include wireless networks, artificial intelligence, machine learning, next generation networks, Internet of Things (IoT) and Mobile/distributed/Cloud Computing. Her research interests are in wireless networks, artificial intelligence, machine learning, next generation networks, Internet of Things (IoT) and Mobile/distributed/Cloud Computing.

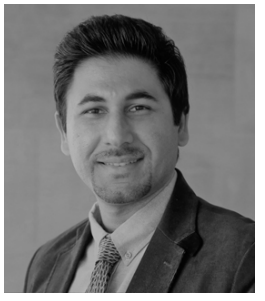

Mohammad Hossein Anisi Mohammad Hossein Anisi (Senior Member, IEEE) is currently an Associate Professor with the School of Computer Science and Electronic Engineering, University of Essex, U.K. and Head of Internet of Everything (IoE) Laboratory. Prior to that, he worked as a Senior Research Associate with the University of East Anglia, U.K. and Senior Lecturer with the University of Malaya, Malaysia, where he received "Excellent Service Award" for his achievements. He has published more than 100 articles in high-quality journals and several conference papers and won two medals for his innovations from PECIPTA 2015 and IIDEX 2016 expositions. His research interests include Internet of Things, Wireless Sensor and Actuator Networks, Green and Energy-efficient Communication and Vehicular Networks. He has received several international and national funding awards for his fundamental and practical research as PI and Co-I. He is an Associate Editor of several journals since 2014. He has been a Guest Editor of special issues of the journals and lead organizer of special sessions and workshops at IEEE conferences such as IEEE ICC, IEEE CAMAD, IEEE PIMRC, and IEEE VTC. He has been also serving as executive/technical committee member of several conferences. $\mathrm{He}$ is fellow of Higher Education Academy.

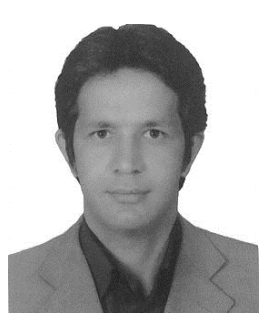

Seyed Ahmad Soleymani received his Ph.D. degree in computer science from faculty of engineering, Universiti Teknologi Malaysia (UTM). He received his M.S degree from the Department of Computer Engineering, Islamic Azad University, Iran and B.S. degree from the Department of Computer Engineering, Sadjad University, Iran. His research interests are in the area of Wireless Sensor Network (WSN), Mobile Ad Hoc Network (MANET), Vehicular Ad Hoc Network (VANET), Internet of Things and Nano Things (IoT and IoNT), Visible Light Communication (VLS), Intelligent Algorithms (IAs), Big Data and Machine Learning.

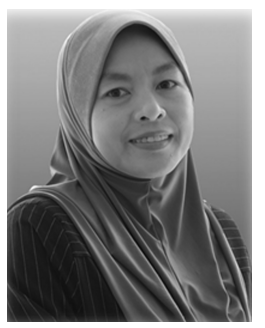

Masri Ayob is a Professor in the Faculty of Information Science and Technology at the Universiti Kebangsaan Malaysia (UKM) since 1997. She has obtained her $\mathrm{PhD}$ in Computer Science at The University of Nottingham in 2005. Her main research areas include meta-heuristics, hyper-heuristics, scheduling and timetabling, especially educational timetabling, healthcare personnel scheduling and routing problems, and Internet of Things. She has published more than 100 papers at international journals and at peerreviewed international conferences. She has been served as a programme committee for more than 50 international conferences and reviewers for high impact journals. She was a member of ASAP research group at the University of Nottingham. Currently, she is a principle researcher in Data Mining and Optimisation Research Group (DMO), Centre for Artificial Intelligent (CAIT), UKM.

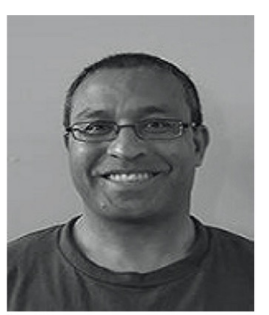

Sherali Zeadally earned his bachelor's degree in computer science from the University of Cambridge, England. He also received a doctoral degree in computer science from the University of Buckingham, England, followed by postdoctoral research at the University of Southern California, Los Angeles, CA $\mathrm{He}$ is currently an Associate Professor in the College of Communication and Information, University of Kentucky. His research interests include Cybersecurity, privacy, Internet of Things, computer networks, and energy-efficient networking. He is a Fellow of the British Computer Society and the Institution of Engineering Technology, England. 